\title{
Divergent in vitro/in vivo responses to drug treatments of highly aggressive NIH-Ras cancer cells: a PET imaging and metabolomics-mass-spectrometry study
}

\author{
Daniela Gaglio ${ }^{1,2,6,8}$, Silvia Valtorta ${ }^{1,2,3,4}$, Marilena Ripamonti ${ }^{1,2}$, Marcella Bonanomi ${ }^{2}$, \\ Chiara Damiani ${ }^{2}$, Sergio Todde ${ }^{5}$, Alfredo Simone Negri ${ }^{6}$, Francesca Sanvito7, Fabrizia \\ Mastroianni ${ }^{2}$, Antonella Di Campli ${ }^{8}$, Gabriele Turacchio ${ }^{8}$, Giuseppe Di Grigoli ${ }^{1,2,3}$, \\ Sara Bellolii ${ }^{1,2,3}$, Alberto Luini ${ }^{8}$, Maria Carla Gilardi ${ }^{1,2}$, Anna Maria Colangelo ${ }^{2,9}$, Lilia \\ Alberghina ${ }^{2,9, *}$, Rosa Maria Moresco ${ }^{2,3,4, *}$ \\ ${ }^{1}$ Institute of Molecular Bioimaging and Physiology, National Research Council (IBFM-CNR), Segrate, Italy \\ ${ }^{2}$ SYSBIO.IT, Centre of Systems Biology, Milano, Italy \\ ${ }^{3}$ Experimental Imaging Center, IRCCS San Raffaele Scientific Institute, Milan, Italy \\ ${ }^{4}$ Department of Medicine and Surgery, University of Milano-Bicocca, Monza, Italy \\ ${ }^{5}$ Tecnomed Foundation of University of Milano-Bicocca, Monza, Italy \\ ${ }^{6}$ Department of Agricultural and Environmental Sciences - Production, Landscape, Agroenergy University of Milan, Milan, \\ Italy \\ ${ }^{7}$ Mouse Histopathology Unit, Department of Pathology, IRCCS San Raffaele Scientific Institute, Milan, Italy \\ ${ }^{8}$ Institute of Protein Biochemistry, National Research Council, Naples, Italy \\ ${ }^{9}$ Department of Biotechnology and Biosciences, University of Milano-Bicocca, Milan, Italy \\ *These authors have contributed equally to this work \\ Correspondence to: Daniela Gaglio, email: daniela.gaglio@ibfm.cnr.it \\ Rosa Maria Moresco, email: moresco.rosamaria@hsr.it \\ Keywords: tumor, metabolic rewiring, PET-imaging, metabolomics-mass-spectrometry, oncogenic-K-ras \\ Received: February 02, $2016 \quad$ Accepted: June 17, $2016 \quad$ Published: July 07, 2016
}

\section{ABSTRACT}

Oncogenic $K$-ras is capable to control tumor growth and progression by rewiring cancer metabolism. In vitro NIH-Ras cells convert glucose to lactate and use glutamine to sustain anabolic processes, but their in vivo environmental adaptation and multiple metabolic pathways activation ability is poorly understood. Here, we show that NIHRas cancer cells and tumors are able to coordinate nutrient utilization to support aggressive cell proliferation and survival. Using PET imaging and metabolomics-mass spectrometry, we identified the activation of multiple metabolic pathways such as: glycolysis, autophagy recycling mechanism, glutamine and serine/glycine metabolism, both under physiological and under stress conditions. Finally, differential responses between in vitro and in vivo systems emphasize the advantageous and uncontrolled nature of the in vivo environment, which has a pivotal role in controlling the responses to therapy.

\section{INTRODUCTION}

Decades of studies and advances in high throughput techniques have identified the complex connection between oncogenic signaling pathways, metabolic reprogramming and increased nutrient uptake in cancer cells. Recently, Hanahan and Weinberg have added a state of "deregulated cellular energetics" to the original hallmarks of cancer, stressing the fact that cellular metabolism is substantially altered during oncogenesis and tumor progression [1]. The enhanced glycolytic activity and impaired oxidative phosphorylation (Warburg effect) characterizing tumors have been known for several years and represent the biological basis of the wide diffusion of $\left[{ }^{18} \mathrm{~F}\right] \mathrm{FDG}$ PET in diagnostic oncology [2]. More recently, it has been demonstrated that cancer cells can employ a large 
number of strategies to regulate cell homeostasis, which depend on different genetic or environmental factors (germline or somatic mutations, tissue vascularization, ongoing treatments, nutrients availability, immune system activity) [3-7]. In fact, activation of never-ending metabolic rewiring strategies tunes cancer cells to the continuous changes in environmental conditions, as it occurs in living organisms, to sustain enhanced growth and survival $[8,9]$. Specifically, the metabolic rewiring shown by cancer cells involves not only the Warburg effect with enhanced glycolysis and high glutamine consumption, but also elevated rates of lipid biosynthesis, maintenance of redox homeostasis and limited levels of autophagy in the first steps of oncogenesis [10-12]. Recent evidence has shown that cancer cells can engage glutamine metabolism enzymes, such as PEP-carboxykinase (PCK2) [13] or pyruvate carboxylase (PC) [14], to develop metabolic adaptation and promote enhanced cell growth when even glucose is limited. In addition, they can activate autophagy when glutamine is inhibited [15]. Furthermore, metabolites, such as ATP, acetyl-CoA (AcCoA), reactive oxygen species (ROS) and enzymes with key role in metabolic regulation may act on different signaling pathways controlling tumor growth [9, 16-19]. However, the resulting effect of several cancer metabolic strategies is an abnormal $\mathrm{NAD}(\mathrm{P}) \mathrm{H} / \mathrm{NAD}(\mathrm{P})$ balance ratio, which appears to promote glutamine utilization by reductive carboxylation to sustain amino acids and lipids synthesis and for ROS quenching [4, 10, 11, 20, 21].

Several findings indicated that mutations in oncogenes like ras and myc control tumor growth and spread by acting also on cell metabolism [22-25]. We have recently demonstrated that NIH3T3 fibroblasts harboring an oncogenic K-ras gene (NIH-Ras-G12V; transformed) [26] are able to decouple glucose and glutamine metabolism to support cancer cell growth [27]. In particular, using this simple tumor cell model, we observed that $K$-ras mutation induced per se a metabolic reprogramming that involved a decoupling of glucose and glutamine metabolism and enabled the efficient utilization of both carbon and nitrogen from glutamine for biosynthetic processes. In addition, it was observed that defects in autophagy were able to compromise survival of cancer cells in nutrient starvation and block tumor growth in allograft models [28, 29]. Moreover, it was demonstrated that activating ras mutations or Ras-effector pathways induced autophagy, which is essential for starvation, survival and tumor growth [29]. Given these in vitro results, oncogenic $K$-ras could drive metabolic reprogramming to develop environmental adaptation and/ or resistance to drug treatments especially in vivo where metabolic conditions are more advantageous. In fact, experiments on cell lines cannot recapitulate the wide heterogeneity of tumors that occurs in in vivo conditions [30]. Therefore, targeting cancer metabolism through both in vitro and in vivo studies represent an attractive investigation strategy, particularly for ras-driven tumors that represent a strong therapeutic challenge.

Here, we used a well-characterized cellular model to investigate the role of simultaneous multiple pathways activation in NIH-Ras cells both in vitro and in vivo xenografts nude mice model. In addition, we examined metabolic changes in response to metabolic target drug treatments.

To address these issues, we took advantage of combined metabolomics (gas-chromatography-mass spectrometry - GC/MS) and in vivo PET imaging ([ $\left.{ }^{18} \mathrm{~F}\right]$ FDG and $\left.\left[{ }^{18} \mathrm{~F}\right] \mathrm{FLT}\right)$ approaches, which had the advantages of: a) detailing, at molecular level and within the context of environmental conditions, genetic regulation, altered kinetic activity of enzymes and changes in metabolic reactions $[31,32]$ and b) imaging macro-parameters (glycolysis and cell proliferation), which represent the end-stage of simultaneous activation or inhibition of different metabolic processes and signaling.

This strategy allowed us to verify whether information derived from PET studies had a sufficient diagnostic power in describing lesion phenotypes under different environmental conditions (in our case, perturbation of glutamine metabolism) and to validate targets for a theranostic approach of $K$-ras mutated cancer.

Our data indicated that gas-chromatography-mass spectrometry and PET imaging techniques allowed a complementary characterization of the NIH-Ras tumor model. Specifically, in vitro NIH-Ras cells were glutamine- and autophagy-dependent and inhibition of both pathways led to cell death. In vivo, NIH-Ras tumors maintained an aggressive phenotype, with high cell proliferation and glucose uptake. Following administration of inhibitors of autophagy and/or of glutaminase, tumors engaged in multiple metabolic processes to overcome the inhibition.

\section{RESULTS}

\section{In vitro NIH-Ras cancer fibroblasts activate multiple pathways to sustain enhanced proliferation}

It is well known that autophagy recycles intracellular components into metabolic pathways to sustain demand for growth and proliferation [33]. In accordance with published data [24, 27-29], oncogenic K-ras could cause multiple pathways activation, such as basal autophagy combined with increased glutamine metabolism, in order to sustain environmental adaptation and aggressive cell proliferation in cancer cells in vivo.

To test the activation of basal autophagy mechanisms in our cellular model, we performed bioimaging analyses (Figure 1). Specifically, we found that NIH-Ras under normal nutrient conditions (4mM glutamine -Gln- and $25 \mathrm{mM}$ glucose) showed both an increased number of 
mitochondria within lysosomes (Figure 1A) and a higher number of lysosomes, as indicated by the lysosomal marker Lamp1 as compared to NIH3T3 (Figure 1B, upper panels) consistent with an increase of autophagy mechanism (Figure 1B, lower panels). These results were confirmed by measurements of fluorescence intensity (98.1 RSU measured in NIH-Ras, as compared to 64.18 RSU in NIH3T3) shown in Supplementary Figure S1A.
To further assess the role of basal autophagy in tumor growth, we performed experiments in response to glutamine starvation $(0.5 \mathrm{mM}$ Gln $) \quad[24,27]$. Nutritional stress for $54 \mathrm{~h}$ induced a remarkable increase of autophagic processes only in transformed cells, as revealed by the enhanced number of mitochondria within lysosomes (Figure 1C) and by the lysosomial marker Lamp1 (Figure 1D). Autophagy activation correlated
A
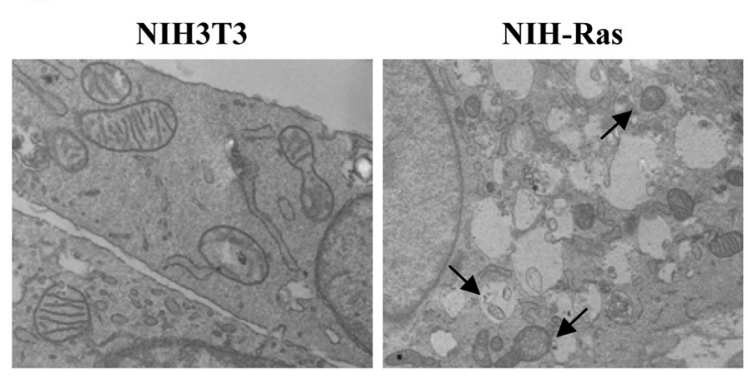

C NIH3T3
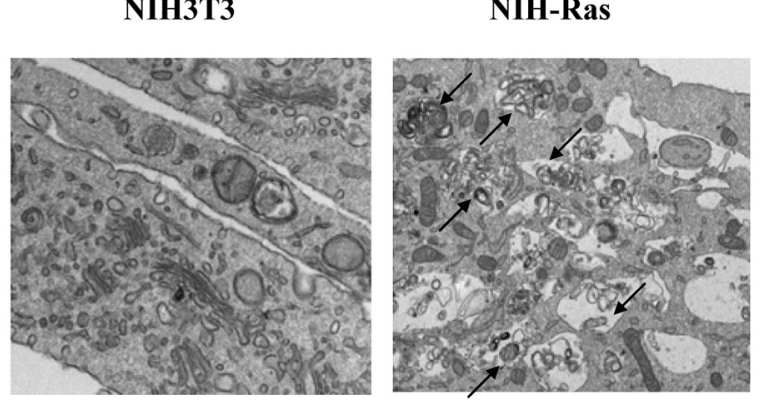

B

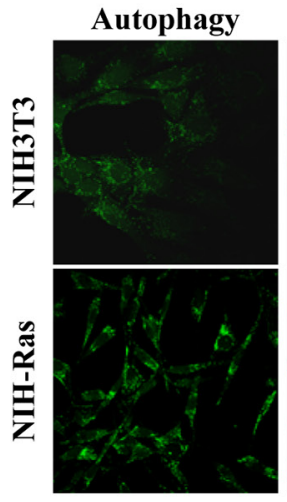

D

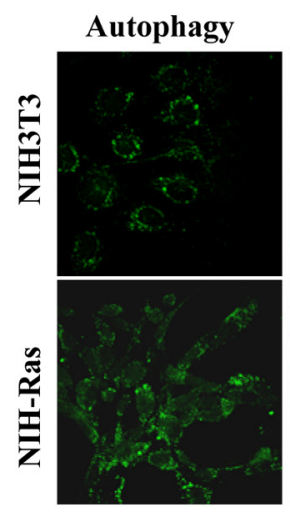

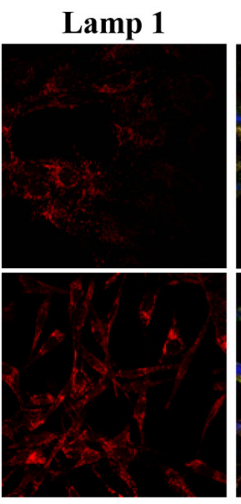
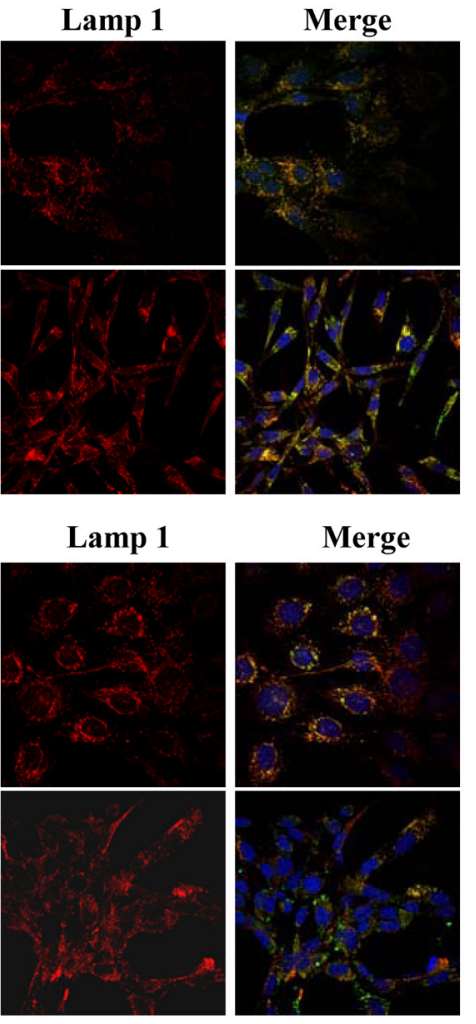

$\square$ NIH3T3

$\mathbf{E}$

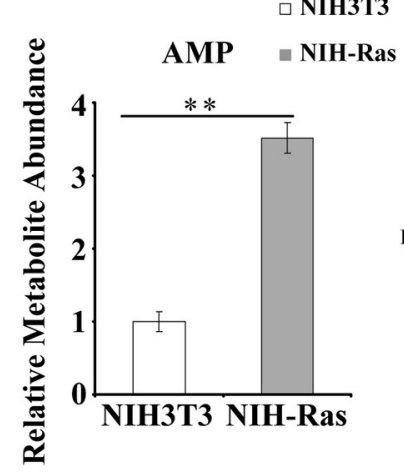

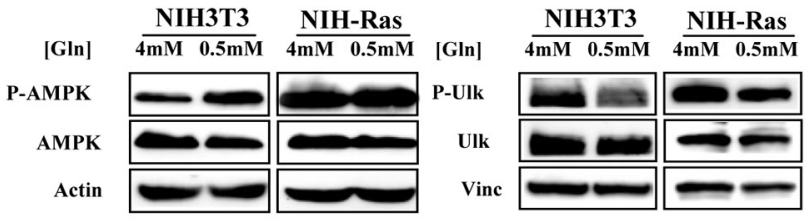

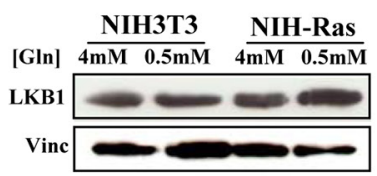

Figure 1: NIH-Ras cancer cells show autophagy activation to sustain enhanced cell proliferation. A, C. Morphological analysis of NIH3T3 and NIH-Ras transformed cells by electron microscopy after 54h under normal growth conditions (4mM Gln) (A) or nutrient deprivation $(0.5 \mathrm{mM}$ Gln) (C); arrows indicate the mitochondria within lysosomes. B, D. Analysis of autophagy and lysosomes organelles in NIH3T3 and NIH-Ras mouse fibroblasts cells under normal growth conditions (4mM Gln) (B) or nutrient deprivation ( $0.5 \mathrm{mM}$ Gln; D) by fluorescence microscopy using Cyto-ID® Autophagy detection kit (green color) and immunostaining for Lamp1 (red color); Hoechst was used for nuclei staining (Blue color). E. Relative AMP abundance in NIH3T3 and NIH-Ras mouse fibroblasts grown under conditions of glutamine deprivation for $54 \mathrm{~h}$. AMP was measured by GC/MS and error bars indicate SD (n=3). F. Protein expression analysis of NIH3T3 and NIH-Ras cell lines grown in media containing $4 \mathrm{mM}$ glutamine (optimal growth condition) or $0.5 \mathrm{mM}$ glutamine (nutrient starvation). Cells were collected $54 \mathrm{~h}$ after treatment and $30 \mu \mathrm{g}$ proteins from the total cellular extracts were processed by SDSPAGE followed by Western blotting with the indicated antibodies. 
with a significant increase of relative AMP abundance (Figure 1E) and increased levels of AMPK, P-AMPK, ULK1, P-ULK1 and LKB1 proteins in NIH-Ras cells both in normal $(4 \mathrm{mM})$ and glutamine deprived conditions (0.5mM Gln), as compared to NIH3T3 cells (Figure 1F and Supplementary Figure S1B-C and S1D).
In addition, the metabolic profiling of NIH-Ras grown under glutamine deprivation displayed increased levels of free amino acids, mainly glutamine (Figure 2A), and TCA cycle metabolites (Figure 2B), suggesting that replacement of metabolites at different levels could be provided via autophagy to ensure nutrient demand under
A

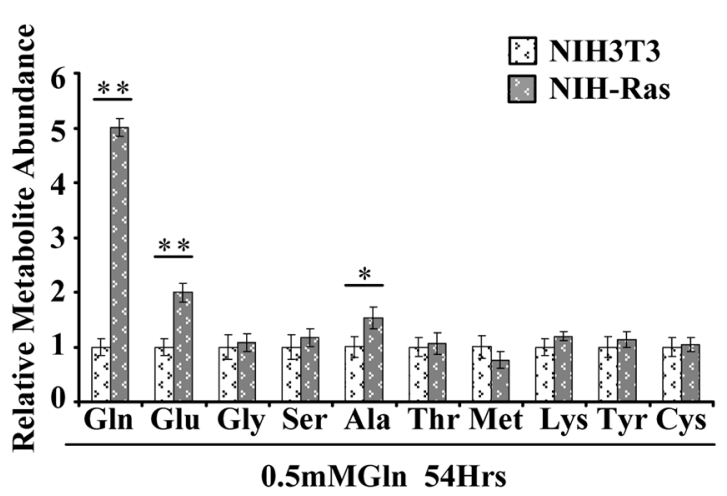

B

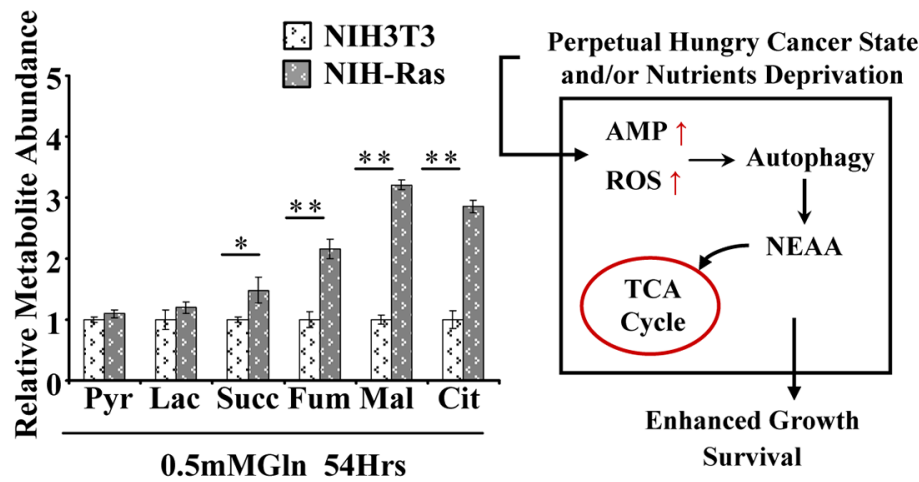

D

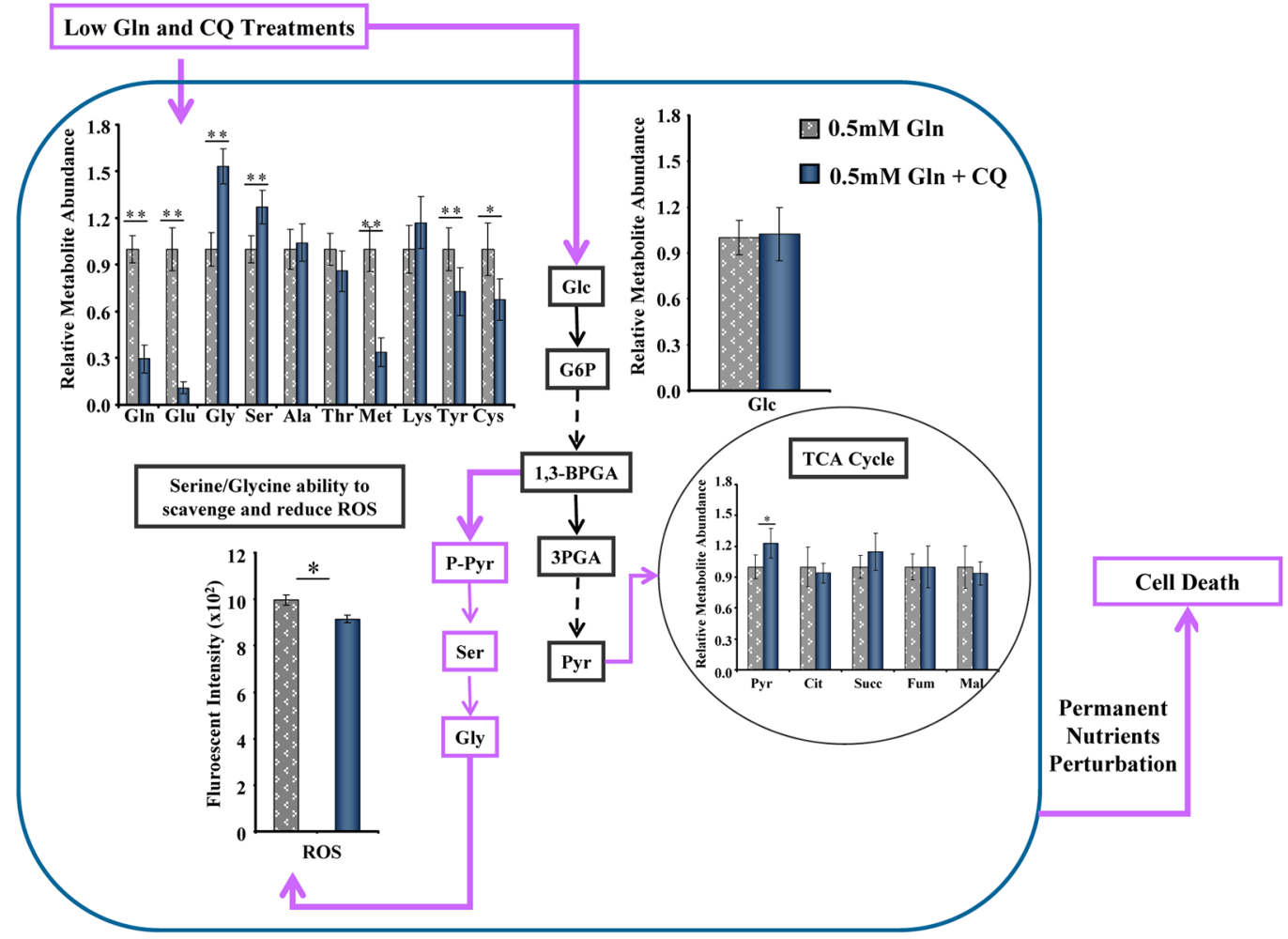

Figure 2: Basal autophagy recycle of metabolites and alternative pathways activation in NIH-Ras cancer cells. A and B. Relative metabolites abundance, free amino acids (A) and TCA cycle metabolites (B), measured by GC/MS in NIH3T3 and NIH-Ras cells cultured in low glutamine $(0.5 \mathrm{mM} \mathrm{Gln})$ for $54 \mathrm{~h}$. C. Schematic representation summarizing how cancer metabolic ability in sustaining permanent nutrient request takes advantage of autophagy mechanisms induced by high ROS and AMP levels to provide amino acids to the TCA cycle. D. Schematic representation of basal autophagy recycle of metabolites and alternative pathways activation. Relative metabolites abundance performed by GC/MS in NIH-Ras cells grown in $0.5 \mathrm{mM}$ Gln or $0.5 \mathrm{mM}$ Gln plus chloroquine $(\mathrm{CQ}, 50 \mu \mathrm{M})$ for $54 \mathrm{~h}$. Error bars indicate SD $(\mathrm{n}=5)$. Intracellular ROS levels measured by DCFDA staining in NIH-Ras grown in medium containing $0.5 \mathrm{mM}$ Gln or $0.5 \mathrm{mM}$ Gln plus CQ for $54 \mathrm{~h}$. Error bars indicate SD ( $\mathrm{n}=3)$. 
stress and satisfy the permanent hungry state (Figure 2C) [29]. As expected, the metabolic profiling of NIHRas under metabolic perturbation with low glutamine plus chloroquine (CQ), an inhibitor of autophagosomal degradation [34, 35] showed a decreased relative abundance of glutamine, glutamate and other amino acids, as compared to low glutamine conditions (Figure 2D).

On the other hand, we also observed significant increased levels of serine (Ser) and glycine (Gly) (Figure 2D), but we did not find significant changes in intracellular relative abundance of glucose and TCA cycle metabolites in NIH-Ras grown in low glutamine or low glutamine plus CQ (Figure 2D). Taken together, these results suggest that the strong metabolic stress due to low glutamine plus CQ (Figure 2D) or CQ treatment alone (Supplementary Figure S2A and S2B) could lead NIH-Ras cells to use glucose (Figures 2D and S2A) in alternative pathways, such as serine/glycine pathway (Figures 2D and S2B) [36], in the attempt to decrease ROS levels (Figure 2D and S2C) and trying (unsuccessfully) to survive (Figure 2D). In fact, as shown in Figure 3, CQ treatment induced cell death in NIH-Ras cells grown both in normal growth medium (4mM Gln) (Figure 3A-3B) (trypan blue positive cells $53 \%$, red diamond) and following glutamine starvation for 54h (Figure 3C-3D) (trypan blue positive cells 97.2\%, red diamond).

However, although autophagy seemed to play an important role in sustaining cancer growth and survival during short glutamine deprivation $(54 \mathrm{~h})$, we observed that this mechanism was not able to maintain survival when the glutamine deprivation was prolonged for $144 \mathrm{~h}$ (Supplementary Figure S2D, left and right panels). Specifically, NIH-Ras transformed cells showed a larger decreased cell number when grown under prolonged nutritional stress (Supplementary Figure S2D, right panel), as compared to cells grown in multi-strata in normal growth conditions (Supplementary Figure S2D, left panel). On the other hand, we did not observe differences in NIH3T3 proliferation (Supplementary Figure S2D, left panel), whose decreased cell number at $144 \mathrm{~h}$ is known to be due to confluence and physiological contactdependent death program (anoikis) [37]. Taken together our results identified the key role of autophagy recycling mechanisms and glutamine metabolism in sustaining anabolic processes, redox balance, cancer survival and aggressive cancer proliferation (Figure $3 \mathrm{E}$ ). To confirm the role of low glutamine on cell proliferation, we assessed the effect of different concentrations of BPTES (bis-2-(5phenylacetamido-1,2,4-thiadiazol-2-yl)ethyl sulfide), an inhibitor of glutaminase activity (Supplementary Figure $\mathrm{S} 2 \mathrm{E}$ ). Consistent with previous results, we found that BPTES $(4,6$ or $8 \mu \mathrm{M})$ reduced NIH-Ras cells proliferation, as compared to CTRL (Supplementary Figure S2E), without changing cell proliferation in NIH3T3. Finally, to obtain a synergic effect we performed a simultaneous perturbation of both pathways (autophagy and glutamine) by using CQ and BPTES together. NIH-Ras cells treated with CQ and BPTES showed a dramatic reduction of proliferation (Figure 3F) with $81 \%$ of trypan blue positive cells (Figure 3F, red diamond), as compared to cells grown.

All together, these data suggest that the combination of BPTES and CQ could be an interesting intervention strategy to be tested in vivo.

\section{PET imaging and metabolomics-mass- spectrometry revealed a time-dependent metabolic shift in NIH-Ras model}

To determine whether the nutrient utilization rewiring observed in vitro reflected the same metabolic dependencies in vivo, we evaluated NIH-Ras cells in a mouse model of xenograft. In particular, we monitored tumor growth (using caliper), glucose metabolism $\left(\left[{ }^{18} \mathrm{~F}\right]\right.$ FDG-PET) and cell proliferation ([ $\left.{ }^{18} \mathrm{~F}\right]$ FLT-PET). First, we characterized in vivo tumor growth using caliper and PET with $\left[{ }^{18} \mathrm{~F}\right] \mathrm{FDG}$ and $\left[{ }^{18} \mathrm{~F}\right] \mathrm{FLT}$ starting when tumors were palpable. Tumor lesions rapidly grew and $100 \%$ of animals showed palpable tumors 7 days after cells injection (Figure 4A). Ten days after cells injection, animals performed PET scan and images showed the presence of glucose-avid and highly proliferative cancer lesions (Figure 4B). Within 15 days tumors reached volumes of $1090 \pm 456 \mathrm{~mm}^{3}$ (Figure 4A) and animals had to be sacrificed to avoid suffering. The rapid growth of NIH-Ras cells observed, probably due to a more advantageous in vivo environment as compared to in vitro conditions, highlighted NIH-Ras immortalized cancer cells as a highly aggressive in vivo model.

The time-course of lesions progression performed in additional groups of animals confirmed the hyperproliferative phenotype that was maximum at 7 days after tumor implantation and remained stable thereafter. On the contrary, an abrupt increase of $\left[{ }^{18} \mathrm{~F}\right] \mathrm{FDG}$ was observed between 7 and 9 days, which remained stable until the $13^{\text {th }}$ day (Figure 4C-4D). As previously observed, tumors rapidly grew in parallel with increased $\left[{ }^{18} \mathrm{~F}\right] \mathrm{FDG}$ or $\left[{ }^{18} \mathrm{~F}\right]$ FLT volumes (Supplementary Figure S3A and S3B); moreover, measurements of lactate dehydrogenase (LDH) activity in tumors collected after mice sacrifice confirmed the presence of Warburg effect (Figure 4E). On the other hand, we also observed a low in vivo hexokinase (HK) activity (Supplementary Figure S3C), consistent with our NIH-Ras cancer cells transcriptomics published data [27], showing lower HK gene expression as compared to normal cells, suggesting ADP-glucokinase (glucosephosphorylating enzyme) as an alternative enzymatic source [38] to support the high glycolytic metabolism and greater advantage in ATP production. Moreover, consistently with the rapid growth and $\left[{ }^{18} \mathrm{~F}\right] \mathrm{FLT}$ uptake, we found increased levels of metabolites involved in nucleotide synthesis (Figures $4 \mathrm{~F}$ and S3D) between day 7 and day 9. Specifically, we observed increased 
relative abundance of adenosine (Aden), adenine (Ade), hypoxanthine (Hpx), xanthine (Xan), GMP and spermidine (Spd) (Figure 4F). Furthermore, we found significant changes in the relative abundance of various amino acids (Figure 4G). Notably, the majority of essential and nonessential amino acids were more abundant at day 9, as compared to day 7 and day 13 (Figure 4G).
To investigate morphological features and to correlate in vivo data with proliferation markers, post mortem H\&E analysis and IHC confirmed the high aggressiveness of NIH-Ras tumors. Samples displayed high mitotic activity regardless of the time points, with only small necrotic areas present mainly at 13 days (Figure $4 \mathrm{H})$. IHC staining revealed tumors with homogeneous and
A

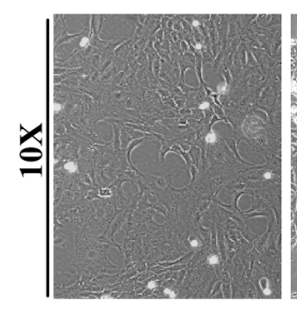

CQ
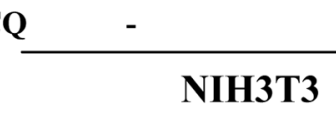

C

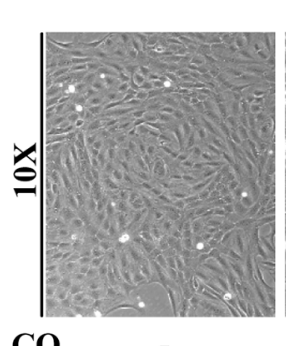

$\mathbf{C Q}$

C

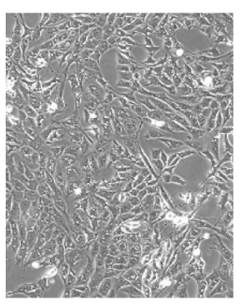

$+$

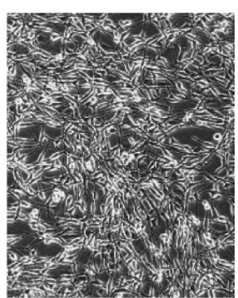

-

NIH-Ras

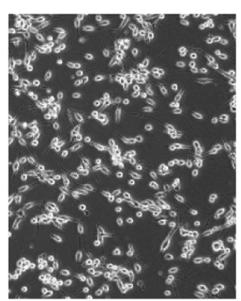

$+$

B

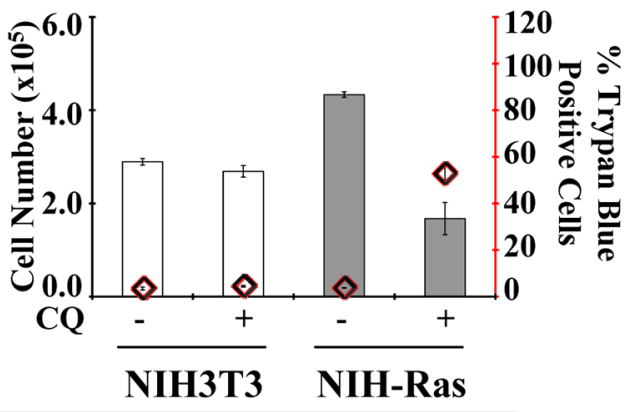

D

4mM Gln

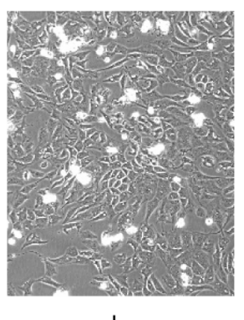

$+$

NIH3T3

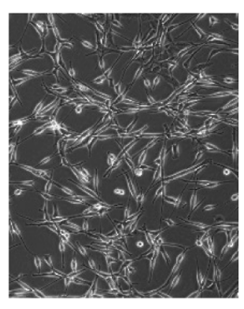

$-$

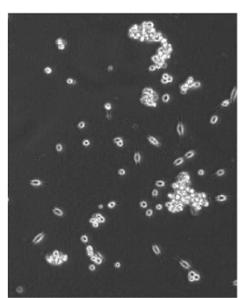

$+$

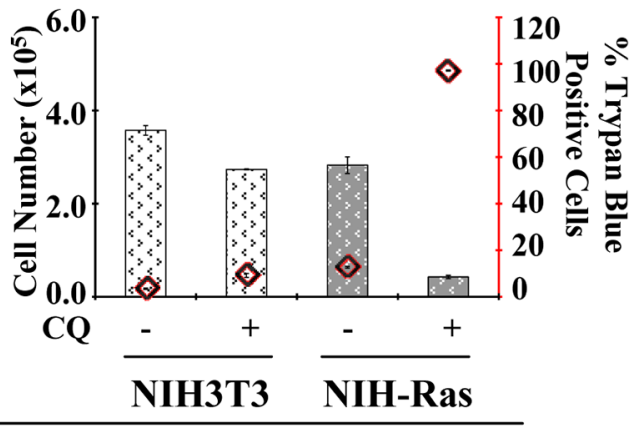

NIH3T3 NIH-Ras

$0.5 \mathrm{mM}$ GIn

$\mathbf{E}$

F
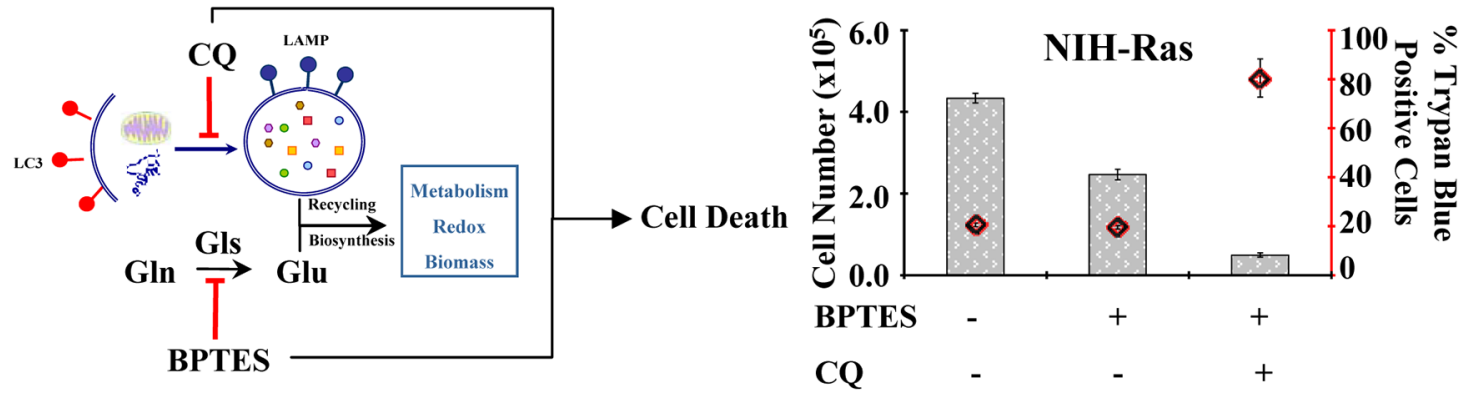

Figure 3: Aggressive proliferative K-Ras transformed fibroblasts show glutamine-dependent metabolic rewiring. A. Morphological analysis of NIH3T3 and NIH-Ras transformed cells cultured in normal growth conditions CTRL or treatment with CQ $(50 \mu \mathrm{M})$ for $24 \mathrm{~h}$. Cells were observed at phase-contrast microscopy. B. Percentage of NIH3T3 and NIH-Ras trypan blue positive cells cultured under normal growth conditions CTRL or after treatment with CQ $(50 \mu \mathrm{M})$ for $24 \mathrm{~h}$. C. Morphological analysis of NIH3T3 and NIH-Ras transformed cells cultured in $0.5 \mathrm{mM}$ Gln CTRL in the absence or presence of CQ $(50 \mu \mathrm{M})$ for $24 \mathrm{~h}$. D. Percentage of normal and transformed trypan blue positive cells cultured in $0.5 \mathrm{mM}$ Gln CTRL in the absence or presence of CQ (50 $\mu \mathrm{M})$ for $24 \mathrm{~h}$. E. Schematic representation of autophagy and glutamine metabolism sustaining cancer growth and their inhibition by BPTES and CQ treatments. F. Proliferation curve of NIH-Ras mouse fibroblasts treated with BPTES, or CQ or their combination. Cells were plated at $3000 / \mathrm{cm}^{2}$. After $18 \mathrm{~h}$ cells were treated with BPTES $(6 \mu \mathrm{M})$ or CQ $(50 \mu \mathrm{M})$ or BPTES+CQ and counted after $54 \mathrm{~h}$. Error bars indicate SD $(\mathrm{n}=3)$. 
A

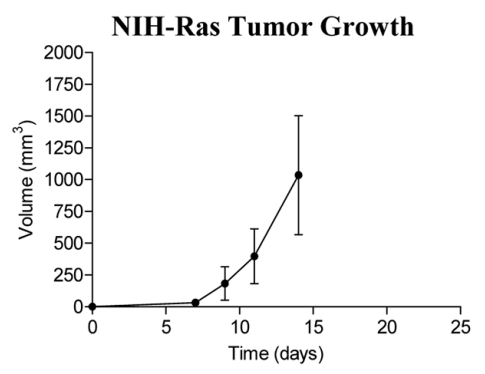

B

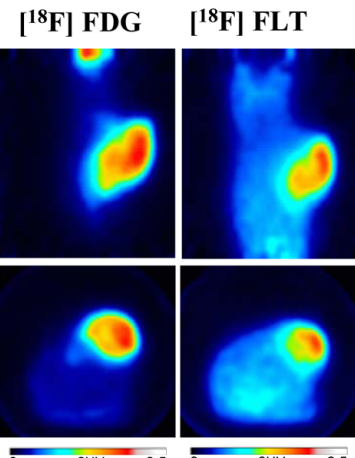

D

Days

7

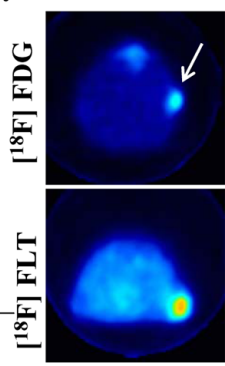

9

13
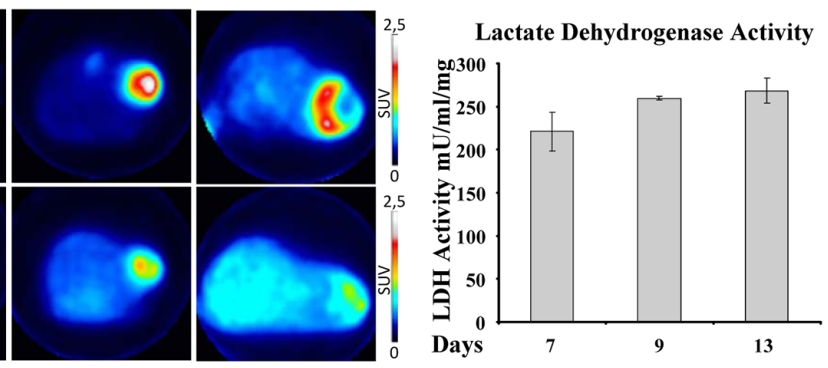

G

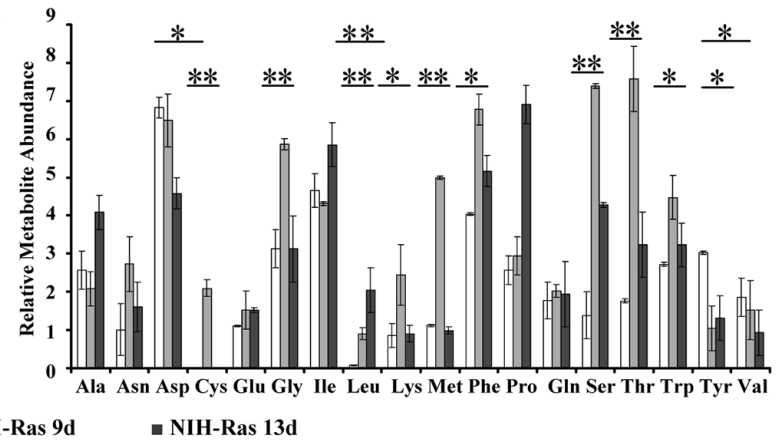

H

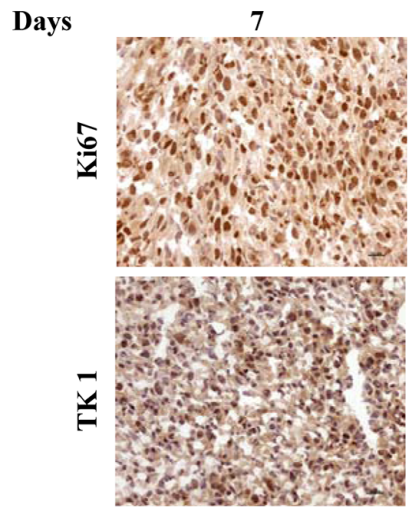

9
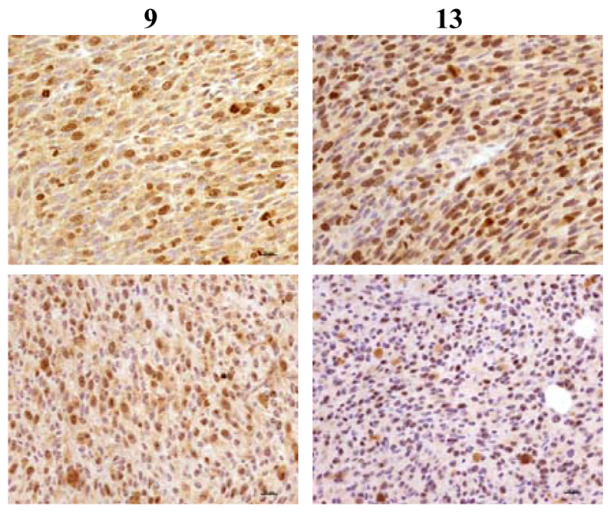

Figure 4: PET imaging and metabolomics technique identify fast growth and highly aggressive phenotype of NIH-Ras tumor xenografts in vivo. A. NIH-Ras tumor xenografts growth curve was established by caliper measurement after s.c. injection of NIH-Ras cancer cell fibroblasts. B. Representative $\left[{ }^{18} \mathrm{~F}\right] \mathrm{FDG}$ and $\left[{ }^{18} \mathrm{~F}\right] \mathrm{FLT}$ PET coronal and transaxial images of NIH-Ras tumors performed at 10 days after cells injection. Color scale is expressed as SUV. C. $\left[{ }^{18} \mathrm{~F}\right] \mathrm{FDG}$ and $\left[{ }^{18} \mathrm{~F}\right] \mathrm{FLT}$ uptake expressed as T/B ratio at different time points in NIH-Ras tumors. From day 7 to day $9\left[{ }^{18} \mathrm{~F}\right] \mathrm{FDG}$ uptake significantly increased $(\mathrm{p}<0.001)$. Error bars indicate SD $(\mathrm{n}=5-8 \mathrm{mice}$ per PET study). D. Representative $\left[{ }^{18} \mathrm{~F}\right] \mathrm{FDG}$ and $\left[{ }^{18} \mathrm{~F}\right] \mathrm{FLT}$ transaxial images of NIH-Ras tumors at different time points from cell injection. Color scale is expressed as SUV and the white arrow indicates the tumor. E. LDH enzyme activity of NIH-Ras tumor xenografts from 7 to 13 days. LDH enzyme activity was measured by enzymatic assay and normalized to protein content. Error bars indicate SD ( $\mathrm{n}=3$ ). $\mathbf{F}$ and G. Relative metabolites abundance involved in nucleotides $(\mathrm{F})$ and amino acids $(\mathrm{G})$ metabolism of NIH-Ras tumor xenografts from 7 to 13 days measured by GC/MS. H. Ki67 and TK1 IHC staining of NIH-Ras tumors collected from mice at 7, 9 and 13 days after cells injection. 
high positivity for both Ki67 and TK-1 antibodies that slowly increased over time (Figure $4 \mathrm{H}$ ), thus confirming $\left[{ }^{18} \mathrm{~F}\right]$ FLT PET data.

Taken together, these results indicated a metabolic shift between 7 to 9 days (glucose uptake and amino-acid levels), which could be a potential therapeutic window to maximize the effects of treatments that target cell metabolism.

\section{Highly aggressive NIH-Ras tumor response to metabolic stress conditions detected by PET imaging and metabolomic-mass-spectrometry}

To evaluate in vivo metabolic changes in drug treatment response and to validate PET imaging and metabolomic-mass-spectrometry as complementary diagnostic tools, mice with NIH-Ras tumors were treated with BPTES and CQ, alone or in combination, using the therapeutic windows previously identified, i.e. at 7 days. In contrast to in vitro results, surprisingly, BPTES alone or in combination with CQ failed to arrest tumor growth (Figure 5A), glucose uptake ([ $\left.\left.{ }^{18} \mathrm{~F}\right] \mathrm{FDG}-\mathrm{PET}\right)$ and cell proliferation ([$\left.\left.{ }^{18} \mathrm{~F}\right] \mathrm{FLT}-\mathrm{PET}\right)$ (Figures $5 \mathrm{~B}$ and $\mathrm{S} 4 \mathrm{~A}-\mathrm{B}$ ). In addition, post mortem data revealed no significant difference in glutamate levels between CTRL and BPTES treated mice (Supplementary Figure S4C) and showed the collateral effect of inducing autophagy (Supplementary Figure S4D) [15].

Consistent with PET findings, metabolomics analysis did not show significant changes either in metabolite composition or in metabolite abundance across all treatments (Supplementary Figure S5A). Hierarchical clustering of samples was performed on normalized intensities of 85 significant metabolites (Supplementary Figure S5A) followed by pathways enrichment of nucleotide metabolism (Supplementary Figure S5B, left panel), glycolysis and TCA cycle (Supplementary Figure $\mathrm{S} 5 \mathrm{~B}$, right panel). The resulting dendrogram, in which the length of the vertical lines connecting two groups of samples is proportional to the distance between them, confirmed the close similarity among groups. Although all groups are similar to each other, CTRL and combined treatment clustered together (Supplementary Figure S5A), whereas BPTES and CQ pertained to a separate cluster. In addition, 6 metabolites ( $\beta$-alanine, urea, glycine, ribose, fructose and glucose) out of 85 were found to be significantly different across samples by using one way ANOVA (Figure 5C). Specifically, we found increased relative abundance of glucose (Glc) and Urea in all treatments, as compared to CTRL (Figure 5C). In addition, we observed significant increased relative abundance of fructose (Fru) and glycine (Gly) in BPTES and BPTES plus CQ treatments, respectively, as compared to CTRL. After comparison of the four groups by ANOVA, t-test was applied in order to find out which metabolites were more significantly expressed in single treatment groups versus CTRL (Supplementary Figure S5C and S5D). In particular, we found significant increased relative abundance of glucose, urea and $\beta$-alanine ( $\beta$-Ala) metabolites in BPTES and CQ treatments versus CTRL (Supplementary Figure S5C and S5D, respectively), suggesting a common metabolic response of NIH-Ras tumors to single drug treatments. At the same time, increased relative abundance of metabolites, such as creatinine ( $\mathrm{Cr}$ ), aspartate (Asp) and fructose (Fru) in BPTES treatment, and increased relative abundance of uric acid and hypoxanthine (Hyp) in CQ were found statistically significant in the two groups, as compared to CTRL (Supplementary Figure S5C and S5D, respectively).

To further highlight differences between treatments, one way Anova analysis (Figure 5D) confirmed BPTES and CQ clustering, as indicated by increased relative abundance
$\mathbf{A}$

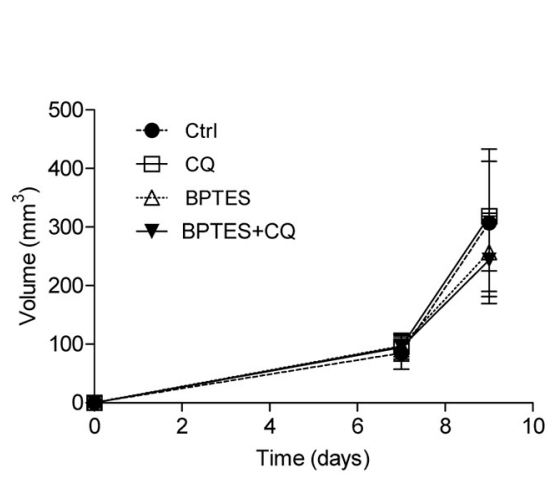

B

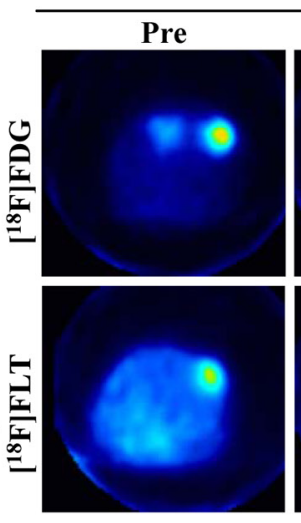

CTRL

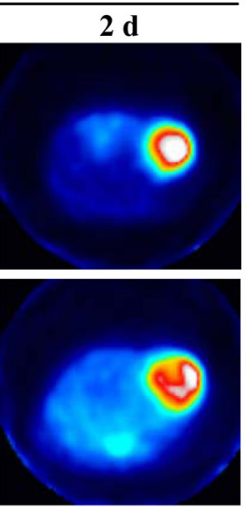

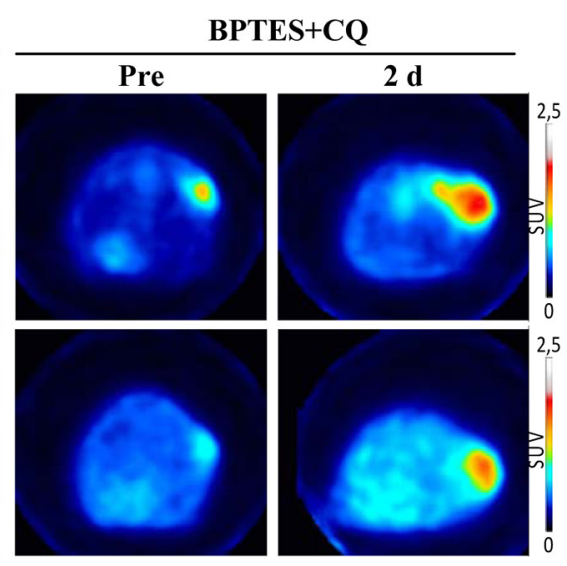

Figure 5: BPTES glutaminase inhibitor and CQ autophagy inhibitor do not show anti-proliferative activity in highly aggressive NIH-Ras tumors. A. Evaluation of tumor size after treatment. Changes in tumor size were measured by caliper in mice treated with a 2-day scheduled treatment. Mice received daily vehicle (CTRL), or CQ (50mg/kg), or BPTES (10 mg/kg), or BPTES plus CQ (Combination). B. Representative transaxial $\left[{ }^{18} \mathrm{~F}\right] \mathrm{FDG}$ and $\left[{ }^{18} \mathrm{~F}\right] \mathrm{FLT}$ PET images of CTRL and combined treatment mice performed before and after drugs administration. Color scale is expressed as SUV value. (Continued) 
C

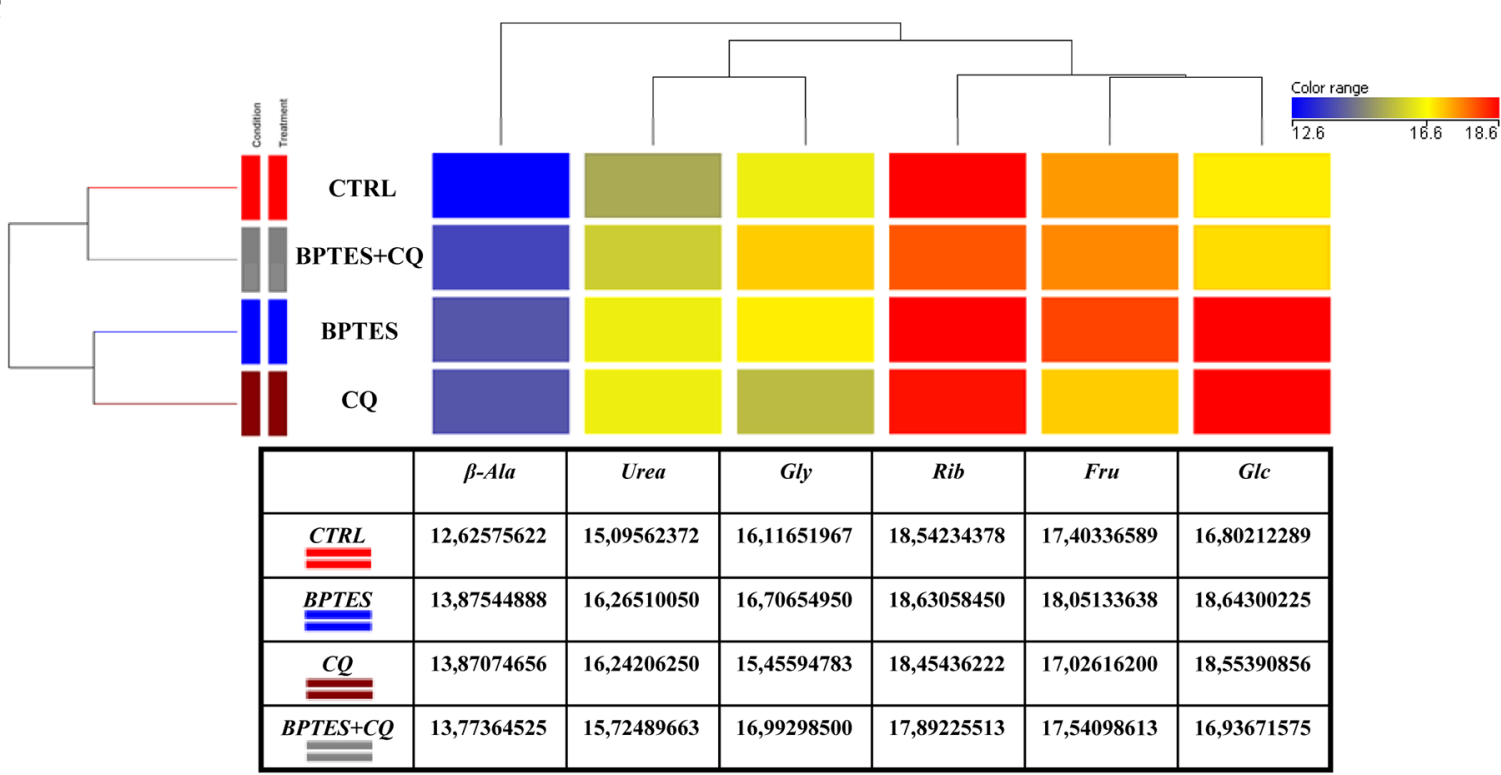

D
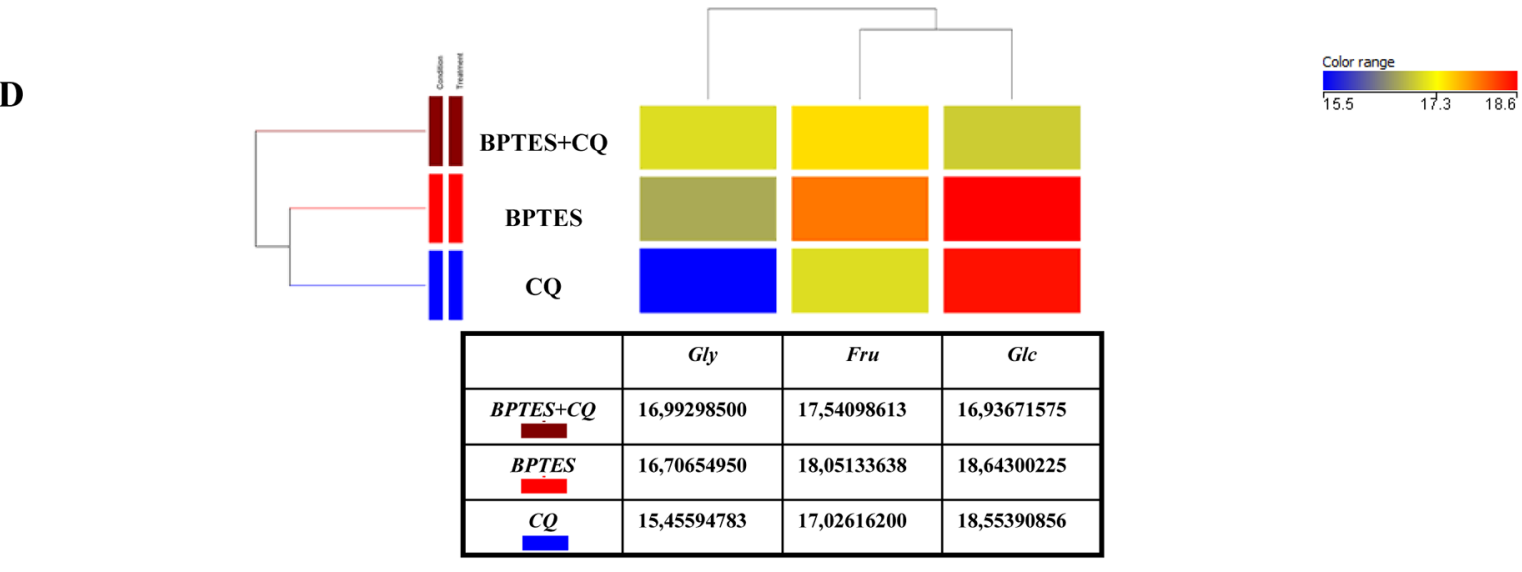

Figure 5: (Continued) BPTES glutaminase inhibitor and CQ autophagy inhibitor do not show anti-proliferative activity in highly aggressive NIH-Ras tumors. C. Comparison of samples by one way ANOVA statistical analysis (CTRL versus single treatments). D. Comparison of samples by one way ANOVA statistical analysis (BPTES and CQ combined treatment versus single treatments). ANOVA statistical analysis was performed using Mass Profiler Professional (MPP) software. The dendrogram was produced by applying a hierarchical clustering algorithm. The color range legends was automatically generated by MPP, considering the minimum and maximum values of most compounds identified to highlight the best differences between samples through the most suitable color scale. Quantitative values of relative metabolites abundance are shown in the tables.

of glucose in both single treatments versus combined treatments, and increased relative abundance of fructose in BPTES alone versus CQ or BPTES and CQ combined treatment (Figure 5D). On the other hand, we found increased relative abundance of glycine in the combined group, as compared to single treatments (Figure 5D). Taken together, these data emphasize that multiple metabolic pathway activation, due to advantageous environmental conditions, could cause the reduced effect of the drugs, at the same time sustaining aggressive cell proliferation of NIH-Ras tumors observed in vivo experiments [39].

\section{DISCUSSION}

Metabolic rewiring following increased nutrients uptake sustains uncontrolled proliferation [6]. In particular, both oncogenic ras and myc stimulate glucose uptake and regulate glutamine metabolism, specifically directing glutamine carbons into pathways able to support biosynthesis, redox homeostasis, cell survival and enhanced growth [27, 40, 41]. A recent work has highlighted the importance of metabolic rewiring in both cultured cells and in vivo mice models [6]. It has been 
demonstrated that glucose deprivation, or growth in the restricted environment of the subcutaneous space in mice, renders transformed K-Ras cancer cells tolerant of low glucose conditions [42]. On the other hand, chronic exposure to low glucose still requires oxidative phosphorylation as a means to maintain growth [43]. Therefore, the complex metabolic requirements of cancer cells lead to greater metabolic flexibility able to activate multiple pathways using alternative substrates that may contribute to ATP synthesis by oxidative phosphorylation (OXPHOS) or intermediate synthesis necessary to sustain anabolic processes for enhanced growth and/or survival $[27,44]$. Hence, tumor cells capable of using alternative substrates for metabolic rewiring modulation in vivo could develop never-ending strategies also under stress conditions.

In this study we used a well characterized rasdriven cancer cell model (NIH-Ras) both in vitro and in vivo to evaluate the role of Ras mutation in cancer lesions response to different environmental conditions using a metabolomics-mass-spectrometry and PET imaging combined approach.

Our results confirmed the glutamine addiction and showed an activation of basal-autophagy in the first steps of cancer cell growth (Figure 1). Under low glutamine condition, we observed an interesting increase of amino acids and TCA cycle metabolites, in particular glutamine, suggesting that autophagy replenishes TCA cycle intermediates to sustain survival (Figure 2) [29]. In agreement with this observation, blocking autophagy by CQ caused a massive cell death, which was further increased by the combined administration of the glutaminase inhibitor (BPTES) (Figure 3). Taken together, these results confirmed that in vitro NIH-Ras cancer cells took advantage not only of enhanced glycolysis, as depicted in the scheme in Figure 6 (red arrows), but also of glutamine metabolism (Figure 6, light-blue arrows) and autophagy recycling (Figure 6, blue arrows) to sustain cellular hyper-proliferation.

Based on these in vitro results confirming that well established multiple pathway activation sustains enhanced growth in NIH-Ras cancer cells, in vivo studies were performed with the purpose to provide a native microenvironment that is metabolically advantageous.

In vivo, NIH-Ras cells gave rise to aggressive lesions characterized by high proliferation rate ([ $\left.\left.{ }^{18} \mathrm{~F}\right] \mathrm{FLT}\right)$ and glucose demand $\left(\left[{ }^{18} \mathrm{~F}\right] \mathrm{FDG}\right)$, which were increased between 7 and 9 days (Figure 4A-4D). Interestingly, in the same time-frame we observed an increase of metabolites involved in nucleotides synthesis (Figure 4F) and augmented relative abundances of various essential and not essential amino acids (Figure 4G). This metabolic shift confirmed that in vivo tumors are able to activate multiple pathways to create a more advantageous environment for cancer growth. In addition, as previously observed in vitro [27], we found reduced activity of HK
(Supplementary Figure S2C), suggestive of the activation ADP-glucokinase, a more efficient hexokinase able to support a higher glycolytic demand [38]. Finally, to evaluate modifications of in vivo metabolism under stress conditions, BPTES and CQ, alone or in combination, were administered to NIH-Ras tumor xenografts. In contrast to our in vitro results, both drugs did not produce notable effects in the rate of lesions growth and PET parameters, particularly when CQ was given alone (Figure 5).

As for BPTES, our results are in line with the observations in BRAF mutated cells displaying high sensitivity to BPTES in vitro but not in vivo [45]. Probably, differently from myc driven lesions, where BPTES was able to suppress tumor growth [42], activation of Ras pathway gives to cancer cells higher ability to sustain tumor growth during metabolic stress.

It is worthy of note that metabolomics analysis showed significant changes in glucose, urea and $\beta$-alanine metabolites when BPTES and CQ were given alone (Figure 5). These results confirmed the key role of glucose (Figure 6, red arrows) and suggest the activation of the urea cycle pathway (Figure 6, yellow arrows) involved in ammonia removal and $\beta$-alanine involved in metabolic rewiring, like it occurs following epithelialmesenchymal transition (EMT) $[39,46]$. On the other hand, the significant increase in glycine levels found under a combined drugs treatment suggests that NIH-Ras tumors could activate multiple alternative pathways, such as the serine-glycine pathway [36], able to sustain the aggressive cell proliferation of NIH-Ras tumors in metabolic stress conditions (Figure 6, lilac arrows) [47, 48]. In such an adaptive system the block of glutaminase activity, as performed by BPTES [49], which spares other glutamine sources, a biosynthesis-dependent process is clearly not sufficient [39].

In addition, in vivo glutamine need is ensured by glutamine producer organs through an inter-organ glutamine trafficking that is modified in cancer to increase glutamine availability. For instance, in a mice model of fibrosarcoma, tumor lesion induces muscle expression of glutamine synthetase and greatly increases the release of glutamine into the circulation [50].

This systemic metabolic rearrangement characterizing cancer might also explain the lack of $\mathrm{CQ}$ effect, together with a progressive loss of autophagy dependence of NIH-Ras cells during tumor progression, even when CQ was administered before the activation of the alternative metabolic pathways, as previously reported [51].

In conclusion, in this study we demonstrated the amazing adaptation ability of ras driven tumors to environmental modifications and metabolic stress using metabolic rewiring and alternative pathways. Differential responses between in vitro and in vivo systems have been shown to be particularly evident under in vivo conditions where nutrients supply is continuously available from 
different body reserves and where probably systemic anabolic processes are also adapted to sustain cancer progression.

These observations may explain why one of the few therapeutic options for ras driven tumors is represented by drugs able to block angiogenesis with a general reduction of nutrient supply. This suggests that general metabolic sensors, which are able to regulate the activation of alternative pathways, should be considered. Finally, we showed that the combined use of PET imaging and metabolomics-mass spectrometry can detail at the molecular level the specific metabolic signature of tumors. This information might be relevant for validating the potential diagnostic power of metabolomics for clinical

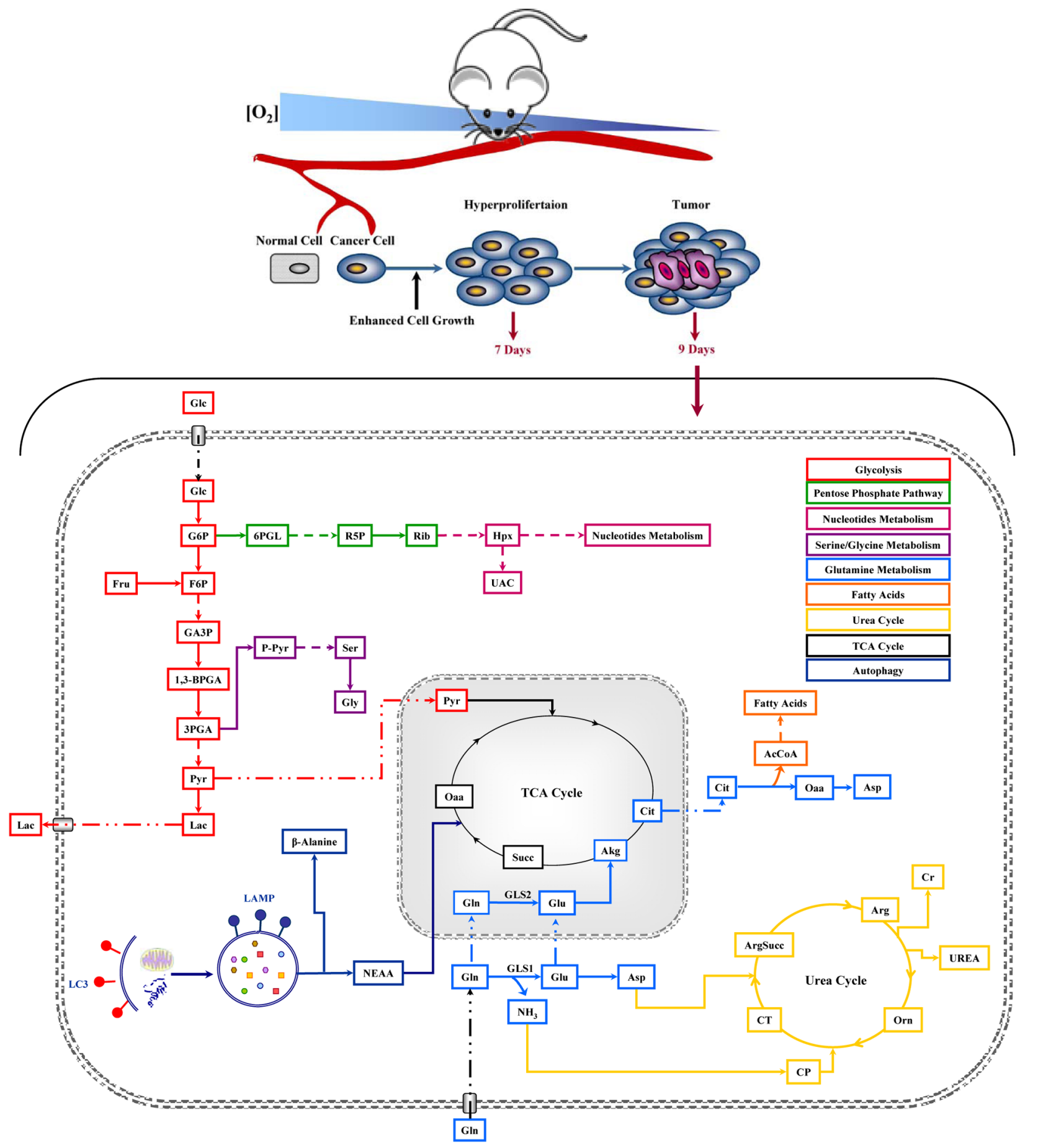

Figure 6: Schematic representation of cancer metabolic pathways identified in in vivo NIH-Ras tumor xenografts and the effect of metabolic interfering drugs. Metabolic Profiling was performed 9 days after cell injection and after $48 \mathrm{~h}$ of drug treatments. 
use in conjunction with the imaging parameters (in this case glycolysis and cell proliferation), which represent the end stage of the simultaneous activation or inhibition of different metabolic processes and signaling.

\section{MATERIALS AND METHODS}

\section{Cell culture}

Mouse embryonic fibroblast NIH3T3 cells (CRL1658; American Type Culture Collection), K-Ras transformed NIH3T3-derived cell line 226.4.1 [52] were routinely grown in Dulbecco's modified Eagle's medium containing $4 \mathrm{mM}$ L-glutamine, $100 \mathrm{U} / \mathrm{ml}$ penicillin and $100 \mathrm{mg} / \mathrm{ml}$ streptomycin (normal growth medium) supplemented with $10 \%$ newborn calf serum at $37^{\circ} \mathrm{C}$ in a humidified atmosphere of 5\% CO2. Cells were passed using trypsin-EDTA and maintained in culture for $54 \mathrm{~h}$ or $144 \mathrm{~h}$ before manipulation. All reagents for media were purchased from Life Technologies Invitrogen (Carlsbad, CA, USA).

\section{Cell proliferation analysis and cell treatments}

Cells were plated at the density of 3000 cells $/ \mathrm{cm}^{2}$ in complete growth medium. After 18h, cells were washed twice with phosphate-buffered saline (PBS) and incubated in a media with different glutamine concentrations $(4 \mathrm{mM}$ or $0.5 \mathrm{mM}$ Gln).

For specific treatments, cells were treated with $50 \mu \mathrm{M}$ Chloroquine (CQ), or 4-6-8 $\mu \mathrm{M}$ BPTES (SigmaAldrich Inc.). To measure cell proliferation, harvested cells were counted by Burker chamber and live/dead count was performed using trypan blue exclusion.

\section{Animal model}

To generate the mouse model, NIH-Ras cells $\left(2.5 \times 10^{5}\right)$ were injected subcutaneously (s.c) on the right flanks of 8-week old nu/nu female mice (Harlan Laboratories). Animals were kept under specific pathogenfree conditions, handled and maintained according to San Raffaele Institutional Animal Care and Use Committee (IACUC) ethical regulations, which previously approved the experiments described in this study. After cells injection, mice were monitored twice a week for body weight and tumor volume was measured using a digital caliper and calculated following the formula: tumor volume $=\left(\right.$ long side $\left.\left.*(\text { short side })^{\wedge} 2\right) / 2\right)$. At the end of studies, mice were sacrificed by $\mathrm{CO}_{2}$ asphyxiation and tumors collected for post mortem analysis.

\section{Study design characterization}

Different groups of animals were evaluated using PET and $\left[{ }^{18} \mathrm{~F}\right] \mathrm{FDG}$ or $\left[{ }^{18} \mathrm{~F}\right] \mathrm{FLT}$ at 7,9 and 13 days after cell injection ( $n=5-8$ per time point and per radiotracer). At the end of PET study, animals were sacrificed and tumors collected for post mortem metabolomics and immunohistochemistry analyses.

\section{Metabolic perturbation by pharmacological therapy}

In vivo studies were performed using BPTES (Bis-2-[5-phenylacetamido-1,2,4-thiadiazol-2-yl]ethyl sulphide, intraperitoneal, i.p. $10 \mathrm{mg} / \mathrm{kg}$ in $2 \% \mathrm{DMSO}$, Sigma-Aldrich $\AA$ ), an inhibitor of glutaminase enzyme and chloroquine (CQ, i.p. $50 \mathrm{mg} / \mathrm{kg}$ in PBS, Sigma-Aldrich), an inhibitor of autophagosomal degradation, alone or in combination. Mice were divided into 4 groups $(n=3-5)$ of treatment (Vehicle, BPTES, CQ and Comb) and treated for 2 consecutive days starting 7 days after cell injection. Control animals received i.p. vehicle. Animals performed $\left[{ }^{18} \mathrm{~F}\right] \mathrm{FDG}$ and $\left[{ }^{18} \mathrm{~F}\right] \mathrm{FLT}$ PET before and after therapy.

\section{PET studies}

PET images were obtained using a YAP-(S)-PET II small animal scanner (ISE s.r.1., Pisa, Italy). For $\left[{ }^{18} \mathrm{~F}\right] \mathrm{FLT}$ studies, 4.2 $\pm 0.3 \mathrm{MBq}$ were injected in the tail vein (i.v.). $\left[{ }^{18} \mathrm{~F}\right] \mathrm{FLT}$ production, acquisition and images quantification were performed as previously described [31]. [ $\left.{ }^{18} \mathrm{~F}\right] \mathrm{FDG}$ was prepared for clinical use (European Pharmacopeia VIII Edition) and i.v. injected (4.3 $\pm 0.2 \mathrm{MBq})$ in animals in fasting conditions. The same procedure of acquisition and image analysis used for $\left[{ }^{18} \mathrm{~F}\right] \mathrm{FLT}$ was applied to $\left[{ }^{18} \mathrm{~F}\right]$ FDG scan. Radioactivity concentrations were expressed as Standardized Uptake Value $($ SUVmax $=(\max$ radioactivity measured in the $\mathrm{ROI} /$ injected radioactivity)*animal weight) and tumor-to-background radioactivity concentration ratios $(\mathrm{T} / \mathrm{B})$.

\section{Post mortem analyses}

For immunohistochemistry (IHC) studies, after sacrifice, cancer lesions were removed, dissected, fixed in $4 \%$ PFA, cryopreserved in liquid $\mathrm{N}_{2}$ cooled isopentane and embedded in OCT. Serial $6 \mu \mathrm{m}$ thick sections were treated with $0.3 \% \mathrm{H}_{2} \mathrm{O}_{2}$ to quench endogenous peroxidase activity. Cell proliferation was evaluated using Ki67 and TK1 antibodies (Novus Biological) to correlate results with $\left[{ }^{18} \mathrm{~F}\right] \mathrm{FLT}$ data.

\section{Enzymatic assays}

For enzymatic assay quantification of LDH Colorimetric Assay kit (BioVision), HK Colorimetric Assay kit (BioVision) and glutamate Colorimetric Assay kit (BioVision), 10mg of NIH Ras tumors were rapidly homogenized on ice in $0.1 \mathrm{ml}$ of appropriate assay buffer and processed according to the manufacturer's protocols. 


\section{ROS levels measurement}

ROS levels were measured using the DCFDA Cellular Ros Detection Assay Kit from Abcam (Cambridge, UK). Cells were harvested and stained with $20 \mu \mathrm{M}$ dichloro-diyidro-fluoresceine-diacetate (DCFDA) for $30 \mathrm{~min}$ at $37^{\circ} \mathrm{C}$. Thereafter, cells were washed and fluorescence was measured at excitation/emission wavelengths of $485 \mathrm{~nm} / 535 \mathrm{~nm}$ respectively using Cary Eclipse Fluorescence Spectrophotometer.

\section{Metabolite profiling cell culture samples}

Metabolites were extracted and analyzed by gas chromatography-mass spectrometry (GC/MS), as described previously [27]. Details are reported in Supplemental Experimental Procedures.

\section{Metabolite profiling in tissue samples}

For tissue metabolite extraction, $0.1 \mathrm{ml}$ ice-cold methanol was added to $10 \mathrm{mg}$ of tissue and incubated $5 \mathrm{~min}$ on ice. An equal volume of water was added and samples were sonicated $5 \mathrm{sec}$ for 5 pulses at $70 \%$ power for three times. After a $30 \mathrm{~min}$ incubation at $-80^{\circ} \mathrm{C}$, samples were sonicated as described above. One volume of chloroform was added and samples were vortexed at $4^{\circ} \mathrm{C}$ for $30 \mathrm{~min}$ and then centrifuged at $12000 \mathrm{~g}$ for $10 \mathrm{~min}$. The aqueous phase was recovered and evaporated under airflow at $37^{\circ} \mathrm{C}$ [53].

Derivatization was performed using automated sample prep WorkBench instrument (Agilent Technologies) as detailed in the Supplemental Experimental Procedures.

Dried polar metabolites were dissolved in $60 \mu \mathrm{l}$ of $2 \%$ methoxyamine hydrochloride in pyridine (Pierce) and held at $40^{\circ} \mathrm{C}$ for $6 \mathrm{~h}$. After dissolution and reaction, $90 \mu \mathrm{l}$ of MSTFA (N-Methyl-N-(trimethylsilyl) trifluoroacetamid) were added and samples were incubated at $60^{\circ} \mathrm{C}$ for $1 \mathrm{~h}$.

GC/MS analysis was performed using 7200 accurate-mass Q-TOF GC/MS (Agilent Technologies) equipped with a 40-m DB-5MS capillary column operating under electron impact (EI) ionization at $70 \mathrm{eV}$. Samples $(2 \mu \mathrm{l})$ were injected in a splitless mode at $250^{\circ} \mathrm{C}$, using helium as the carrier gas at a flow rate of $1 \mathrm{ml} / \mathrm{min}$. The GC oven temperature was held at $100^{\circ} \mathrm{C}$ for $2 \mathrm{~min}$ and increased to $325^{\circ} \mathrm{C}$ at $10^{\circ} \mathrm{C} / \mathrm{min}$.

GC/MS data processing was performed using Agilent Muss Hunter software and statistical analyses were performed using Mass Profile Professional (MPP) software [54]. Relative metabolites abundance was carried out after normalization to internal standard norvaline and cell number.

\section{Electron microscopy}

Electron microscopy experiments were performed as described in Supplemental Experimental Procedures [55].

\section{Immunofluorescence microscopy}

Protocols and antibodies are described in the Supplemental Experimental Procedures.

\section{Western blotting analysis}

Protocols and antibodies are described in the Supplemental Experimental Procedures.

\section{Statistical analysis}

Results are expressed as mean value \pm SD. Experimental differences were tested for significance with the Student's $t$-test or, when possible, with the One Way ANOVA test. A p-value of 0.05 or less was considered statistically significant.

\section{ACKNOWLEDGMENTS}

This work was supported by grants from the SysBioNet project, a MIUR initiative for the Italian Roadmap of European Strategy Forum on Research Infrastructures (ESFRI), to L.A., M.B., C.D., F.M., G.D., A.M.C. We thank I. Raccagni, P Simonelli, and E.A. Turolla of University of Milano-Bicocca and M Rocchi of Mouse Histopathology Unit, Department of Pathology, IRCCS San Raffaele Scientific Institute for technical support. The Authors wish to thank Agilent Technologies team for technical suggestions and Rohit Bharat for suggestions on the manuscript.

\section{CONFLICTS OF INTEREST}

The authors declare that they have no conflict of interest.

\section{REFERENCES}

1. Hanahan D and Weinberg RA. Hallmarks of cancer: the next generation. Cell. 2011; 144:646-674.

2. Warburg O. On the origin of cancer cells. Science (New York, NY). 1956; 123:309-314.

3. Quail DF and Joyce JA. Microenvironmental regulation of tumor progression and metastasis. Nature medicine. 2013; 19:1423-1437.

4. Alberghina L and Gaglio D. Redox control of glutamine utilization in cancer. Cell death \& disease. 2014; 5:e1561.

5. Gorrini C, Harris IS and Mak TW. Modulation of oxidative stress as an anticancer strategy. Nat Rev Drug Discov. 2013; 12:931-947.

6. Boroughs LK and DeBerardinis RJ. Metabolic pathways promoting cancer cell survival and growth. Nature cell biology. 2015; 17:351-359.

7. Pavlova NN and Thompson CB. The Emerging Hallmarks of Cancer Metabolism. Cell metabolism. 2016; 23:27-47. 
8. Munoz-Pinedo C, El Mjiyad N and Ricci JE. Cancer metabolism: current perspectives and future directions. Cell death \& disease. 2012; 3:e248.

9. Galluzzi L, Kepp O, Vander Heiden MG and Kroemer G. Metabolic targets for cancer therapy. Nat Rev Drug Discov. 2013; 12:829-846.

10. Schulze A and Harris AL. How cancer metabolism is tuned for proliferation and vulnerable to disruption. Nature. 2012; 491:364-373.

11. Vander Heiden MG, Cantley LC and Thompson CB. Understanding the Warburg effect: the metabolic requirements of cell proliferation. Science (New York, NY). 2009; 324:1029-1033.

12. White E. Deconvoluting the context-dependent role for autophagy in cancer. Nature reviews. 2012; 12:401-410.

13. Vincent EE, Sergushichev A, Griss T, Gingras MC, Samborska B, Ntimbane T, Coelho PP, Blagih J, Raissi TC, Choiniere L, Bridon G, Loginicheva E, Flynn BR, Thomas EC, Tavare JM, Avizonis D, et al. Mitochondrial Phosphoenolpyruvate Carboxykinase Regulates Metabolic Adaptation and Enables Glucose-Independent Tumor Growth. Molecular cell. 2015; 60:195-207.

14. Sellers K, Fox MP, Bousamra M, 2nd, Slone SP, Higashi RM, Miller DM, Wang Y, Yan J, Yuneva MO, Deshpande R, Lane AN and Fan TW. Pyruvate carboxylase is critical for non-small-cell lung cancer proliferation. The Journal of clinical investigation. 2015; 125:687-698. doi: 10.18632/ oncotarget.2173.

15. Lee YZ, Yang CW, Chang HY, Hsu HY, Chen IS, Chang HS, Lee CH, Lee JC, Kumar CR, Qiu YQ, Chao YS and Lee SJ. Discovery of selective inhibitors of Glutaminase-2, which inhibit mTORC1, activate autophagy and inhibit proliferation in cancer cells. Oncotarget. 2014; 5:60876101. doi:10.18632/oncotarget.2173.

16. Locasale JW and Cantley LC. Metabolic flux and the regulation of mammalian cell growth. Cell metabolism. 2011; 14:443-451.

17. Luo W and Semenza GL. Pyruvate kinase M2 regulates glucose metabolism by functioning as a coactivator for hypoxia-inducible factor 1 in cancer cells. Oncotarget. 2011; 2:551-556. doi: 10.18632/oncotarget.299.

18. Wellen $\mathrm{KE}$ and Thompson $\mathrm{CB}$. A two-way street: reciprocal regulation of metabolism and signalling. Nat Rev Mol Cell Biol. 2012; 13:270-276.

19. Yang W, Xia Y, Ji H, Zheng Y, Liang J, Huang W, Gao X, Aldape K and Lu Z. Nuclear PKM2 regulates beta-catenin transactivation upon EGFR activation. Nature. 2011; 480:118-122.

20. Daye D and Wellen KE. Metabolic reprogramming in cancer: unraveling the role of glutamine in tumorigenesis. Seminars in cell \& developmental biology. 2012; 23:362-369.

21. Hirschey MD, DeBerardinis RJ, Diehl AM, Drew JE, Frezza C, Green MF, Jones LW, Ko YH, Le A, Lea MA,
Locasale JW, Longo VD, Lyssiotis CA, McDonnell E, Mehrmohamadi M, Michelotti G, et al. Dysregulated metabolism contributes to oncogenesis. Seminars in cancer biology. 2015; 35:S129-150.

22. Chiaradonna F, Gaglio D, Vanoni M and Alberghina L. Expression of transforming K-Ras oncogene affects mitochondrial function and morphology in mouse fibroblasts. Biochimica et biophysica acta. 2006; 1757:1338-1356.

23. Chiaradonna F, Sacco E, Manzoni R, Giorgio M, Vanoni $\mathrm{M}$ and Alberghina L. Ras-dependent carbon metabolism and transformation in mouse fibroblasts. Oncogene. 2006; 25:5391-5404.

24. Gaglio D, Soldati C, Vanoni M, Alberghina L and Chiaradonna F. Glutamine deprivation induces abortive s-phase rescued by deoxyribonucleotides in k-ras transformed fibroblasts. PloS one. 2009; 4:e4715.

25. Yuneva M, Zamboni N, Oefner P, Sachidanandam R and Lazebnik Y. Deficiency in glutamine but not glucose induces MYC-dependent apoptosis in human cells. The Journal of cell biology. 2007; 178:93-105.

26. Bossu P, Vanoni M, Wanke V, Cesaroni MP, Tropea F, Melillo G, Asti C, Porzio S, Ruggiero P, Di Cioccio V, Maurizi G, Ciabini A and Alberghina L. A dominant negative RAS-specific guanine nucleotide exchange factor reverses neoplastic phenotype in K-ras transformed mouse fibroblasts. Oncogene. 2000; 19:2147-2154.

27. Gaglio D, Metallo CM, Gameiro PA, Hiller K, Danna LS, Balestrieri C, Alberghina L, Stephanopoulos G and Chiaradonna F. Oncogenic K-Ras decouples glucose and glutamine metabolism to support cancer cell growth. Molecular systems biology. 2011; 7:523.

28. Guo JY, Chen HY, Mathew R, Fan J, Strohecker AM, Karsli-Uzunbas G, Kamphorst JJ, Chen G, Lemons JM, Karantza V, Coller HA, Dipaola RS, Gelinas C, Rabinowitz JD and White E. Activated Ras requires autophagy to maintain oxidative metabolism and tumorigenesis. Genes \& development. 2011; 25:460-470.

29. Mathew R and White E. Autophagy, stress, and cancer metabolism: what doesn't kill you makes you stronger. Cold Spring Harbor symposia on quantitative biology. 2011; 76:389-396.

30. Rosa R, Monteleone F, Zambrano N and Bianco R. In vitro and in vivo models for analysis of resistance to anticancer molecular therapies. Current medicinal chemistry. 2014; 21:1595-1606.

31. Curtarello M, Zulato E, Nardo G, Valtorta S, Guzzo G, Rossi E, Esposito G, Msaki A, Pasto A, Rasola A, Persano L, Ciccarese F, Bertorelle R, Todde S, Plebani M, Schroer H, et al. VEGF-targeted therapy stably modulates the glycolytic phenotype of tumor cells. Cancer research. 2014; 75:120-133.

32. Sauter AW, Wehrl HF, Kolb A, Judenhofer MS and Pichler BJ. Combined PET/MRI: one step further in multimodality imaging. Trends in molecular medicine. 2010; 16:508-515. 
33. White E. The role for autophagy in cancer. The Journal of clinical investigation. 2015; 125:42-46.

34. Fan C, Wang W, Zhao B, Zhang S and Miao J. Chloroquine inhibits cell growth and induces cell death in A549 lung cancer cells. Bioorganic \& medicinal chemistry. 2006; $14: 3218-3222$.

35. Jiang PD, Zhao YL, Deng XQ, Mao YQ, Shi W, Tang QQ, Li ZG, Zheng YZ, Yang SY and Wei YQ. Antitumor and antimetastatic activities of chloroquine diphosphate in a murine model of breast cancer. Biomedicine \& pharmacotherapy. 2010; 64:609-614.

36. Pacold ME, Brimacombe KR, Chan SH, Rohde JM, Lewis CA, Swier LJ, Possemato R, Chen WW, Sullivan LB, Fiske BP, Cho S, Freinkman E, Birsoy K, Abu-Remaileh M, Shaul YD, Liu CM, et al. A PHGDH inhibitor reveals coordination of serine synthesis and one-carbon unit fate. Nature chemical biology. 2016.

37. Taddei ML, Giannoni E, Fiaschi T and Chiarugi P. Anoikis: an emerging hallmark in health and diseases. The Journal of pathology. 2012; 226:380-393.

38. Ronimus RS and Morgan HW. Cloning and biochemical characterization of a novel mouse ADP-dependent glucokinase. Biochemical and biophysical research communications. 2004; 315:652-658.

39. Davidson SM, Papagiannakopoulos T, Olenchock BA, Heyman JE, Keibler MA, Luengo A, Bauer MR, Jha AK, O'Brien JP, Pierce KA, Gui DY, Sullivan LB, Wasylenko TM, Subbaraj L, Chin CR, Stephanopolous G, et al. Environment Impacts the Metabolic Dependencies of RasDriven Non-Small Cell Lung Cancer. Cell metabolism. 2016; 23:517-528.

40. Son J, Lyssiotis CA, Ying H, Wang X, Hua S, Ligorio M, Perera RM, Ferrone CR, Mullarky E, Shyh-Chang N, Kang Y, Fleming JB, Bardeesy N, Asara JM, Haigis MC, DePinho $\mathrm{RA}$, et al. Glutamine supports pancreatic cancer growth through a KRAS-regulated metabolic pathway. Nature. 2013; 496:101-105.

41. Weinberg F, Hamanaka R, Wheaton WW, Weinberg S, Joseph J, Lopez M, Kalyanaraman B, Mutlu GM, Budinger GR and Chandel NS. Mitochondrial metabolism and ROS generation are essential for Kras-mediated tumorigenicity. Proceedings of the National Academy of Sciences of the United States of America. 2010; 107:8788-8793.

42. Le A, Lane AN, Hamaker M, Bose S, Gouw A, Barbi J, Tsukamoto T, Rojas CJ, Slusher BS, Zhang H, Zimmerman LJ, Liebler DC, Slebos RJ, Lorkiewicz PK, Higashi RM, Fan TW, et al. Glucose-independent glutamine metabolism via TCA cycling for proliferation and survival in B cells. Cell metabolism. 2012; 15:110-121.

43. Birsoy K, Possemato R, Lorbeer FK, Bayraktar EC, Thiru P, Yucel B, Wang T, Chen WW, Clish CB and Sabatini DM. Metabolic determinants of cancer cell sensitivity to glucose limitation and biguanides. Nature. 2014; 508:108-112.
44. Gross MI, Demo SD, Dennison JB, Chen L, ChernovRogan T, Goyal B, Janes JR, Laidig GJ, Lewis ER, Li J, Mackinnon AL, Parlati F, Rodriguez ML, Shwonek PJ, Sjogren EB, Stanton TF, et al. Antitumor activity of the glutaminase inhibitor CB-839 in triple-negative breast cancer. Molecular cancer therapeutics. 2014; 13:890-901.

45. Baenke F, Chaneton B, Smith M, Van Den Broek N, Hogan K, Tang H, Viros A, Martin M, Galbraith L, Girotti MR, Dhomen N, Gottlieb E and Marais R. Resistance to BRAF inhibitors induces glutamine dependency in melanoma cells. Molecular oncology. 2015.

46. Bhowmik SK, Ramirez-Pena E, Arnold JM, Putluri V, Sphyris N, Michailidis G, Putluri N, Ambs S, Sreekumar A and Mani SA. EMT-Induced Metabolite Signature Identifies Poor Clinical Outcome. Oncotarget. 2015; 6:42651-60. doi: 10.18632/oncotarget.4765.

47. Amelio I, Cutruzzola F, Antonov A, Agostini M and Melino G. Serine and glycine metabolism in cancer. Trends in biochemical sciences. 2014; 39:191-198.

48. Jain M, Nilsson R, Sharma S, Madhusudhan N, Kitami T, Souza AL, Kafri R, Kirschner MW, Clish CB and Mootha VK. Metabolite profiling identifies a key role for glycine in rapid cancer cell proliferation. Science (New York, NY). 2012; 336:1040-1044.

49. Yuneva MO, Fan TW, Allen TD, Higashi RM, Ferraris DV, Tsukamoto T, Mates JM, Alonso FJ, Wang C, Seo Y, Chen $\mathrm{X}$ and Bishop JM. The metabolic profile of tumors depends on both the responsible genetic lesion and tissue type. Cell metabolism. 2012; 15:157-170.

50. Hensley CT, Wasti AT and DeBerardinis RJ. Glutamine and cancer: cell biology, physiology, and clinical opportunities. The Journal of clinical investigation. 2013; 123:3678-3684.

51. Morgan MJ, Gamez G, Menke C, Hernandez A, Thorburn J, Gidan F, Staskiewicz L, Morgan S, Cummings C, Maycotte $\mathrm{P}$ and Thorburn A. Regulation of autophagy and chloroquine sensitivity by oncogenic RAS in vitro is context-dependent. Autophagy. 2014; 10:1814-1826.

52. Pulciani S, Santos E, Long LK, Sorrentino V and Barbacid M. ras gene Amplification and malignant transformation. Molecular and cellular biology. 1985; 5:2836-2841.

53. Tian Y, Xu T, Huang J, Zhang L, Xu S, Xiong B, Wang $\mathrm{Y}$ and Tang H. Tissue Metabonomic Phenotyping for Diagnosis and Prognosis of Human Colorectal Cancer. Scientific reports. 2016; 6:20790.

54. Musharraf SG, Mazhar S, Choudhary MI, Rizi N and Atta ur R. Plasma metabolite profiling and chemometric analyses of lung cancer along with three controls through gas chromatography-mass spectrometry. Scientific reports. 2015; 5:8607.

55. Filadi R, Greotti E, Turacchio G, Luini A, Pozzan T and Pizzo P. Presenilin 2 Modulates Endoplasmic ReticulumMitochondria Coupling by Tuning the Antagonistic Effect of Mitofusin 2. Cell reports. 2016. 